\title{
Interaction Between DUSP8 and the Polyglutamine Protein Ataxin-1
}

\author{
Do Hee Lee ${ }^{\dagger}$ and Sayeon Cho* \\ College of Pharmacy, Chung-Ang University, Seoul 156-756, Korea. *E-mail: sycho@cau.ac.kr \\ 'Department of Biotechnology, College of Natural Science, Seoul Women's University, Seoul 159-744, Korea \\ Received March 4, 2013, Accepted March 26, 2013
}

Key Words : Polyglutamine proteins, Ataxin-1, Protein tyrosine phosphatase, DUSP8

Polyglutamine (polyQ) diseases are a group of inherited neurodegenerative disorders caused by the expansion of polyQ repeats in the disease proteins. Expansion of polyQ repeats presumably leads to misfolding and aggregation of polyQ proteins. ${ }^{1}$ Although details of the molecular mechanisms are still elusive, transcriptional dysfunction and oxidative damage are suggested as underlying events responsible for polyQ pathogenesis. ${ }^{2}$ Misfolded polyQ proteins are frequently concentrated into intranuclear inclusions, which increase generation of reactive oxygen species (ROS) and activate stress signaling pathways. In cultured cells expressing expanded polyQ proteins, mitogen-activated protein kinases (MAPK), e.g., p38 and JNK, are activated and the cytotoxicity induced by polyQ proteins, partially through sustained activation of MAP kinases, can be reduced by administration of a p38 inhibitor. ${ }^{3-6}$ MAPK are activated by MAPK kinase (MEK)-mediated phosphorylation of threonine and tyrosine residues located in the signature sequence (TxY) and MEK is in turn activated by MAPK kinase kinase $(\mathrm{MEKK}){ }^{7}$ Since MAPK are involved in a wide variety of cellular processes including cell survival and apoptosis, cells must tightly regulate the magnitude and duration of MAPK activation. The negative regulation of MAPK is often achieved by phosphatase-mediated dephosphorylation of MAPK. A group of protein phosphatases referred as type I cysteinebased protein tyrosine phosphatases including DUSPs (dualspecificity phosphatases) dephosphorylate both tyrosine and serine/threonine residues within the same substrate and as a result counteract MAPK. ${ }^{8}$

Involvement of protein tyrosine phosphatases (PTPs) in polyQ-induced cell death has been reported in previous studies. Several PTP proteins including MKP-1 are up-regulated by over-expression of cytotoxic polyQ-expanded huntingtin (Htt) fragment. ${ }^{9}$ Moreover, polyQ-expanded Htt fragment activates JNK pathway by reducing solubility of JNK phosphatase M3/6 (also known as hVH5 or DUSP8). ${ }^{4}$ A recent study demonstrated that a DUSP protein laforin can suppress the cytotoxicity caused by misfolded proteins and hinted that phosphatases can be potential targets for pharmacological intervention of neurodegenerative disorders. ${ }^{10}$ The polyQ protein ataxin-1 is a soluble protein of about 816 amino acids, which varies depending on the length of polyQ repeats, and located both in the cytoplasm and nucleus. Although the exact functions are not completely understood, ataxin-1 is apparently involved in transcription regulation through its ability to interact with several transcription factors as well as RNA. ${ }^{11}$ While it is generally assumed that expansion of polyQ repeats causes misfolding of ataxin-1 and leads to neuronal death in spinocerebellar ataxia 1 (SCA1), contribution of other domains to pathogenesis also becomes increasingly clear. Besides N-terminal polyQ region, AXH domain (570-689; for interaction with transcription factors and RNA), endogenous phosphorylation site (776) and nuclear localization signal (795-798) are present in the Cterminus of ataxin- $1 .{ }^{11}$ Phosphorylation of ataxin-1 is particularly important since it is implicated in pathogenicity of SCA1. Phosphorylation at Ser776 is critical for the interaction of ataxin-1 with 14-3-3 proteins that regulate its entry into the nucleus. ${ }^{12}$

Previously we demonstrated that ataxin-1, capable of activating JNK by itself, does not directly interact with this MAPK. ${ }^{5}$ Such observations led us to suspect that misfolded polyQ proteins, while not directly binding to $\mathrm{JNK}$, could physically associate with PTPs (e.g., DUSP proteins) and thereby regulate JNK activation. To explore this possibility, we first investigated if ataxin-1 actually interacts with MAPK phosphatases in vivo. Upon screening for PTPs that physically interact with normal ataxin-1[30Q], we found out that a number of human PTPs - including DUSP2, 8, 13 and 24 strongly associated with ataxin-1[30Q] (Table 1).

Table 1. Interaction between ataxin- $1[30 \mathrm{Q}]$ and protein tyrosine phosphatases (PTPs) in vivo ${ }^{a}$

\begin{tabular}{cccc}
\hline PTP & $\begin{array}{c}\text { Interaction with } \\
\text { Ataxin-1[30Q] }\end{array}$ & PTP & $\begin{array}{c}\text { Interaction with } \\
\text { Ataxin-1[30Q] }\end{array}$ \\
\hline DUSP1 & - & DUSP12 & - \\
DUSP2 & ++ & DUSP13 & ++ \\
DUSP3 & - & DUSP15 & - \\
DUSP4 & + & DUSP24 & ++ \\
DUSP6 & + & MTM1 & - \\
DUSP7 & +- & CDC14B & - \\
DUSP8 & ++ & PTPRH & - \\
DUSP11 & + & & \\
\hline
\end{tabular}

${ }^{a}$ HEK293T cells were transiently co-transfected with FLAG-tagged ataxin-1[30Q] and GST-tagged PTPs. After $48 \mathrm{~h}$ of transfection, cells were lysed and then subjected to immunoprecipitation with anti-FLAG M2 affinity gel followed by western-blot analysis with anti-GST antibody. All data are representative of three independent experiments. $++=$ strong interaction; $+=$ moderate interaction; $+/-=$ inconsistent interaction; $-=$ no interaction. 
(a)
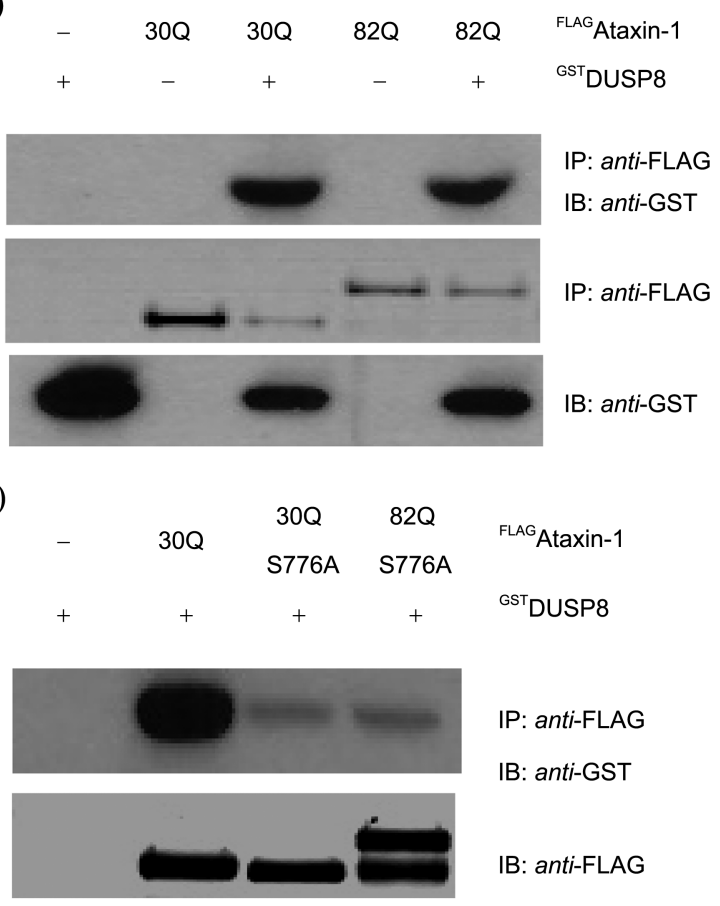

IP: anti-FLAG

IB: anti-GST

IB: anti-FLAG

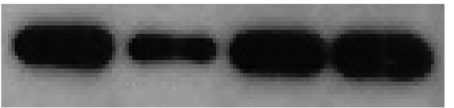

IB: anti-GST

Figure 1. Interaction between DUSP8 and ataxin-1. HEK293T cells were transiently transfected with (a) FLAG-tagged ataxin1[30Q], ataxin-1[82Q] or (b) ataxin-1 mutant (S776A) together with GST-tagged DUSP8. After $48 \mathrm{~h}$ of transfection, cells were collected and lysed with NP40 lysis buffer. Ataxin-1 proteins in cell lysate were isolated using anti-FLAG M2 affinity gels and the presence of ataxin- 1 and DUSP- 8 in the immunoprecipitates was determined by western-blot analysis using anti-FLAG antibody and anti-GST antibody, respectively.

Among them, we were particularly interested in DUSP8 because it belongs to JNK/p38-selective group of MAPK phosphatases and the solubility of DUSP8 (M3/6) was also reported to be impaired by expanded polyQ proteins, causing JNK activation. ${ }^{4,7}$ To further investigate the interaction between DUSP8 and ataxin-1, we then examined if the length of polyQ repeat influences their association. Coimmunoprecipitation experiments were carried out using both normal ataxin-1[30Q] and polyQ-expanded ataxin1[82Q] together with DUSP8. As shown in Figure 1(a), DUSP8 associated with both ataxin-1[30Q] and ataxin$1[82 \mathrm{Q}]$ in a similar extent indicating that polyQ length does not influence the binding and their association is presumably governed by the region(s) outside the polyQ repeats. Indeed interaction analysis using a mutant form of ataxin-1(S776A), in which a major endogenous phosphorylation site (Ser776) is substituted with alanine, revealed that ataxin-1's binding to DUSP8 was impaired by the mutation of this phosphorylation site (Fig. 1(b)).

Since the functional relationship of oxidative stress in polyQ pathogenesis and JNK activation was already welldocumented, we then examined if oxidative stress affects the

\begin{tabular}{|c|c|c|c|c|c|c|c|c|}
\hline${ }^{\mathrm{FLAG}}$ Ataxin-1 & $-30 Q$ & $82 Q$ & $30 Q$ & $82 Q$ & $-30 Q$ & $82 Q$ & $30 Q$ & $82 Q$ \\
\hline${ }^{\text {GST} D U S P 8 ~}$ & $+\quad+$ & + & + & + & + & + & + & + \\
\hline $1 \mathrm{mM} \mathrm{H}_{2} \mathrm{O}_{2}$ & & & + & + & & & + & + \\
\hline
\end{tabular}

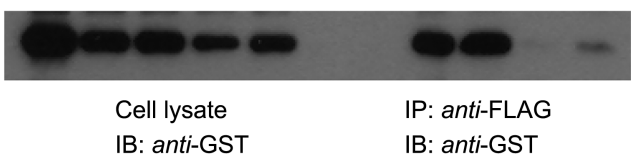

Figure 2. Effects of oxidative stress on the interaction between DUSP8 and ataxin- 1 . After transfection with ataxin- 1 and DUSP8, cells were treated with or without $1 \mathrm{mM} \mathrm{H}_{2} \mathrm{O}_{2}$ for $24 \mathrm{~h}$. Coimmunoprecipitation using anti-FLAG M2 affinity gels was carried out as in Fig. 1.

interaction between DUSP8 and ataxin-1. As expected, the treatment of cells with $1 \mathrm{mM} \mathrm{H} \mathrm{H}_{2} \mathrm{O}_{2}$ nearly abolished the interaction of ataxin-1 and DUSP8 (Fig. 2). Above findings together suggest that the segment near $\mathrm{C}$-terminus of ataxin1 which contains a major phosphorylation site, but not polyQ repeats, is important for the interaction with MAPK phosphatase and their interaction is strongly influenced by the cellular state of oxidative stress.

It was previously demonstrated that DUSP8, even though its solubility was impaired by polyQ proteins, was not directly recruited into nuclear inclusions. ${ }^{4}$ Analysis of domain structure has revealed that DUSP8 contains nuclear export signal (NES) but not nuclear localization signal (NLS). ${ }^{7}$ These results led us to predict that the interaction between DUSP8 and ataxin-1 takes place mainly in cytoplasm but not likely in the nucleus. Confocal microscopic analysis indeed

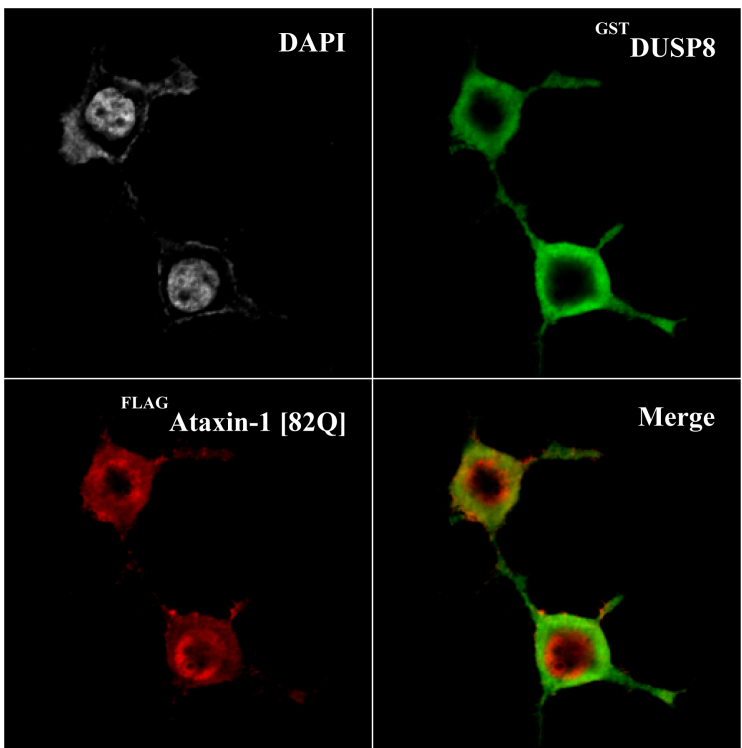

Figure 3. Confocal analysis of interaction between ataxin-1 and DUSP8. For immunofluorescence analysis, HEK293T cells were seeded onto glass slide and then transfected with FLAG-tagged ataxin-1 and GST-tagged DUSP8. After $48 \mathrm{~h}$ of transfection, cells were fixed and then incubated with anti-FLAG antibody and antiGST antibody followed by incubation with Alexa Fluor-conjugated secondary antibodies. DAPI was used for nuclei staining. Magnification $=100 \mathrm{X}$. 
confirmed that DUSP8 and ataxin-1[82Q] were co-localized primarily in the cytoplasm and revealed that DUSP8 was not recruited into the nuclear inclusions containing ataxin1[82Q] (Fig. 3).

Under our experimental conditions, the majority of ataxin1 is phosphorylated in vivo since the phosphorylated form of ataxin- 1 is readily detected in cultured cells even in the absence of external stimuli. ${ }^{13}$ Our findings that the phosphorylation state of ataxin-1 influences interaction between ataxin1 and DUSP8 and the association takes place predominantly in cytoplasm suggest a role of DUSP8 in nuclear transport. Binding of ataxin-1 to certain subtypes of 14-3-3 proteins, which recognize and associate with many phosphoproteins inside cells, is reported to prevent de-phosphorylation and thereby stabilize ataxin- $1 .{ }^{12}$ Whether DUSP8 actually promotes de-phosphorylation of ataxin-1 in vivo is yet to be determined; however, it can be speculated that 14-3-3 proteins and DUSP8 competitively bind to the phosphorylated form of ataxin-1.

Possibly prolonged interaction with DUSP8, which contains nuclear export signal, not only blocks the nuclear translocation but also causes de-stabilization and the eventual degradation of ataxin-1. DUSP8 (M3/6) belongs to JNK/p38selective group of MAPK phosphatases and is inactivated by protein damaging stresses including oxidative stress and heat shock. ${ }^{7}$ Upon heat shock, DUSP8 dissociates from JNK and then becomes inactivated as its solubility is reduced. In addition, DUSP8 is relatively unstable and has an apparent halflife of $2 \mathrm{~h}$ in cultured cells. ${ }^{14,15}$ Reportedly arsenite-induced oxidative stress modulates interaction of DUSP8 with JNK isoforms differentially, which is mediated by modification of DUSP8 (e.g., inactivation by ROS) rather than that of JNK proteins. ${ }^{16}$ Our finding that oxidative stress negatively regulates the association between ataxin-1 and DUSP8 suggests a similar possibility of DUSP8 modification. In fact, we also observed that the presence of ataxin-1 and oxidative stress reduced the relative amount of GST-DUSP8 substantially (Fig. 1 and 2; see anti-GST blot). In polyQ diseases, persistent oxidative stress may perturb physical interaction between polyQ proteins and DUSP8, which under normal circumstances prevents unnecessary entry of misfolded polyQ proteins into the nucleus and subsequent aggregation.

Members of DUSP subfamily, through their ability to negatively regulate MAP kinase signaling cascades, are now recognized as important controllers of many cellular processes. Hence controlling of DUSP activity could provide tools to intervene certain human diseases including neurodegenerative disorders. Recently, efforts to identify selective inhibitors of DUSP have been attempted. Structural analysis of several DUSP proteins will allow us better understanding of molecular architecture and help design more specific inhibitors for DUSP proteins. ${ }^{17}$ However, the physiological roles of individual DUSP proteins are still poorly understood and some of DUSP proteins may have additional functions, such as regulation of protein-protein interaction. Further studies on molecular details of how certain PTPs recognize misfolded proteins and affect their normal functions or localization probably will open up opportunities to utilize this class of PTPs as therapeutic targets for inherited neurodegenerative diseases including Huntington's disease and spinocerebellar ataxia and to develop small molecule inhibitors specific for PTPs.

\section{Experimental}

Cell Culture and DNA Transfection. Human embryonic kidney (HEK) $293 \mathrm{~T}$ cells were maintained at $37{ }^{\circ} \mathrm{C}$ in DMEM supplemented with $10 \%$ fetal bovine serum and penicillin/ streptomycin in the presence of $5 \% \mathrm{CO}_{2}$. FLAG-tagged ataxin1 expression plasmid was constructed in pFLAG/CMV-2 mammalian expression vector and GST-tagged DUSP8 was constructed in $\mathrm{pEBG}$ expression vector, respectively. For transient transfection, $1.4 \times 10^{6}$ cells were plated in $60-\mathrm{mm}$ cell culture plates, grown overnight, and transfected with various plasmid DNA using Lipofectamine reagent (Invitrogen).

Immunoprecipitation and Western-blot Analysis. After $48 \mathrm{~h}$ of expression of FLAG-tagged ataxin-1 with GST-tagged PTP constructs (for oxidative stress experiments, cells were treated with $1 \mathrm{mM}$ hydrogen peroxide for $24 \mathrm{~h}$ ), HEK293T cells were collected and then lysed in NP-40 lysis buffer $(20 \mathrm{mM}$ Tris-HCl, pH 8.0, $150 \mathrm{mM} \mathrm{NaCl}, 1 \mathrm{mM}$ EDTA, 0.5\% NP-40) supplemented with Complete-MINI ${ }^{\mathrm{TM}}$ protease inhibitor mixture (Roche) for $20 \mathrm{~min}$ at $4{ }^{\circ} \mathrm{C}$, followed by centrifugation at $13,000 \times g$ for $20 \mathrm{~min}$. For coimmunoprecipitation, the cell lysates were incubated with 20 $\mu \mathrm{L}$ of anti-FLAG M2 agarose (Sigma) at $4{ }^{\circ} \mathrm{C}$ for overnight with rotation. After washing with phosphate buffered saline (PBS), the beads were resuspended in 1x SDS sample buffer and boiled for $10 \mathrm{~min}$. Western-blot analysis using anti-GST antibody $(1: 1,000$; Santa Cruz) or anti-FLAG antibody (1:1,000; Sigma) was performed to detect individual proteins. The protein bands were visualized using ECL detection system (PIERCE).

Confocal Microscopy Analysis. HEK293T cells were cultured to confluence and then seeded onto glass slide (1-2 $\times 10^{5}$ cells). After $48 \mathrm{~h}$ of transfection with FLAG-tagged ataxin-1 and GST-tagged DUSP8, the cells were washed in 1 $\times \mathrm{PBS} / 3.7 \%$ formaldehyde for $15 \mathrm{~min}$ and then twice in $1 \times$ $\mathrm{PBS} / 0.1 \%$ triton $\mathrm{X}-100$ for $15 \mathrm{~min}$ at room temperature. After Image-i $\mathrm{T}^{\mathrm{TM}} \mathrm{FX}$ signal enhance (Molecular Probe) treatment, the slides were incubated with primary antibodies (1:100-1:200) for 1-2 h. After washing twice in $1 \times$ PBS, slides were incubated with Alexa Fluor ${ }^{\circledR} 488$ goat anti-rabbit IgG (for DUSP8) or Alexa Fluor ${ }^{\circledR} 546$ goat anti-mouse IgG (for ataxin-1) for $1 \mathrm{~h}$. The stained cells were observed under a laser confocal fluorescence microscope (Carl Zeiss). For nuclei staining, cells were incubated with $1 \times \mathrm{DAPI} / \mathrm{PBS}$ for $10 \mathrm{~min}$ at room temperature.

Acknowledgments. We are grateful to Dr. Joohyun Ryu (The Hormel Institute, MN, USA) for his valuable assistance and support. This study was supported by Basic Science Research Program through the National Research Founda- 
tion of Korea (NRF) funded by the Ministry of Education, Science and Technology (grant number 2010-0006080).

\section{References}

1. Shao, C.; Diamond, M. I. Hum. Mol. Genet. 2007, 16, R115.

2. Williams, A. J.; Paulson, H. L. Trends in Neurosci. 2008, 31, 521.

3. Cowan, K. J.; Diamond, M. I.; Welch, W. J. Hum. Mol. Genet. 2003, 12,1377

4. Merienne, K.; Helmlinger, D.; Perkin, G. R.; Devys, D.; Trottier, Y. J. Biol. Chem. 2003, 278, 16957.

5. Ryu, J.; Cho, S.; Park, B. C.; Lee, D. H. Biochem. Biophys. Res. Commun. 2010, 393, 280.

6. Tsirigotis, M.; Baldwin, R. M.; Tang, M. Y.; Lorimer, I. A.; Gray, D. A. PLoS One 2008, 3, e2130.

7. Dickinson, R. J.; Keyse, S. M. J. Cell Sci. 2006, 119, 4607.
8. Patterson, K. I.; Brummer, T.; O'Brien, P. M.; Daly, R. J. Biochem. J. 2009, 418,475

9. Wu, Z. L.; O'Kane, T. M.; Scott, R. W.; Savage, M. J.; BozyczkoCoyne, D. J. Biol. Chem. 2002, 277, 44208.

10. Garyali, P.; Siwach, P.; Singh, P. K.; Puri, R.; Mittal, S.; Sengupta, S.; Parihar, R.; Ganesh, S. Hum. Mol. Genet. 2009, 18, 688.

11. Matilla-Dueñas, A.; Goold, R.; Giunti, P. Cerebellum 2008, 7, 106.

12. Orr, H. T. J. Cell Biol. 2012, 197, 167.

13. Jorgensen, N. D.; Andresen, J. M.; Pitt, J. E.; Swenson, M. A.; Zoghbi, H. Y.; Orr, H. T. J. Neurochem. 2007, 102, 2040.

14. Palacios. C.; Collins, M. K.; Perkins, G. R. Curr. Biol. 2001, 11, 1439.

15. Theodosious, A.; Ashworth, A. Oncogene 2002, 21, 2387.

16. Oehrl, W.; Cotsiki, M.; Panayotou, G. Cell Signal. 2013, 25, 429.

17. Jeffrey, K. L.; Camps, M.; Rommel, C.; Mackay, C. R. Nat. Rev. Drug Discov. 2007, 6, 391. 